\title{
Análise numérica da mistura do óleo de Pinhão-Manso e etanol em microcanais
}

\author{
H. S. Santana e O. P. Taranto \\ Universidade Estadual de Campinas, Faculdade de Engenharia Quimica \\ E-mail para contato: harrison.santana@gmail.com
}

\begin{abstract}
RESUMO - Recentemente, grandes avanços vêm sendo realizados na área de miniaturização de sistemas. Esses microdispositivos têm sido aplicados com sucesso na síntese de biodiesel, devido principalmente ao aumento da conversão dos reagentes em um curto tempo de residência. Um dos aspectos mais importantes desses microdispositivos é a mistura das espécies reagentes, que irá determinar a conversão no produto desejado. Em virtude disso, esse trabalho estudou numericamente a mistura de fluidos em microcanais para a produção de biodiesel. Foram estudados três tipos de micromisturadores: forma-T, duplo-T e em forma de cruz. A eficiência de cada tipo foi analisada em função do grau de mistura que é calculado usando a variação da fração mássica. Todos os misturadores apresentaram excelente grau de mistura para baixo números de Reynolds, com o em forma de Cruz mostrando o mais alto grau de mistura. $\mathrm{O}$ aumento do número de Reynolds elevou a mistura no $\mathrm{T}$, reduziu no duploT e não alterou no micromisturador em forma de Cruz.
\end{abstract}

\section{INTRODUÇÃO}

Dispositivos microfluídicos têm revolucionado vários campos do conhecimento, permitindo que sejam realizadas reações, análises químicas, biológicas, médicas e desenvolvimento de novos produtos e processos de forma mais rápida (Goulet e Kjeang, 2014). Dispositivos ou sistemas microfluídicos referem-se aos sistemas com comprimento característico na faixa de micrômetros (Mansur et al., 2008). Sistemas com essa faixa de tamanho permitem utilizar quantidades menores de reagentes e amostras, com menores tempos de residências e análises, maior controle da concentração das espécies químicas no microdispositivo e elevada razão superfície-volume (Whitesides, 2006). Outra característica importante presente em microescala é a dominância das forças viscosas sobre as inerciais.

Essa dominância caracteriza o escoamento de fluidos em microescala como laminar, o que pode em alguns casos dificultar a mistura das espécies. Essa dificuldade é devido ao fato da mistura dos fluidos, na maioria dos micromisturadores, ser resultado principalmente da difusão molecular (Nguyen e Wereley, 2006). Como muitos sistemas necessitam misturar dois ou mais fluidos, micromisturadores são um dos elementos mais importantes dos microdispositivos. Para aumentar a eficiência da mistura, pesquisadores vêm investigando a mistura em microescala e desenvolvendo diferentes tipos de micromisturadores, que funcionam sob diferentes princípios. De forma geral, eles podem ser classificados como ativos e passivos. Os ativos, utilizam uma fonte externa de energia, que pode ser elétrica ou magnética, para realizar a mistura dos fluidos. Já nos passivos não é utilizado nenhuma fonte de energia, além da pressão básica utilizada para guiar o escoamento dos fluidos (Nguyen e Wereley, 2006; Alam et al., 2013). 


\section{9 a 22 de outubro de 2014 \\ Florianópolis/SC}

Os estudos sobre micromisturadores estão se concentrando mais nos misturadores passivos, uma vez que esses requerem um processo mais fácil de fabricação, controle e integração com outros dispositivos, quando comparado aos ativos. Entretanto, misturadores que não necessitam de fonte externa de energia, apresentam um comprimento de mistura e um número de Reynolds maior. Então é de fundamental importância, o desenvolvimento de micromisturadores que apresentem elevada eficiência de mistura em canais menores e em baixos números de Reynols.

Aumentando a superfície de contato entre os fluidos e diminuindo o caminho de difusão entre eles, pode-se elevar a difusão molecular (Nguyen e Wu, 2005). Uma das maneiras de reduzir o caminho de mistura e aumentar a superfície de contato é dividir a corrente de entrada em $n$ subcorrentes, rearranjando elas posteriormente em uma corrente. $\mathrm{Na}$ literatura, isso é chamado de Parallel lamination. O mais básico é um canal com duas entradas $(n=2)$, também chamado de misturador-T. Também pode se reduzir o caminho de difusão pela Parallel lamination utilizando o conceito de hydrodynamic focusing. Os micromisturadores que se utilizam desse conceito, normalmente possuem um canal com três entradas $(n=3)$. No caso de dois fluidos (A e B), enquanto o fluido A escoa pela entrada do meio, o fluido B escoa pelas outras duas entradas. No artigo de revisão de Nguyen e Wu (2005) encontra-se várias utilizações desses micromisturadores.

Esses tipos de micromisturadores vêm sendo utilizados em vários tipos de análises e processos. Uma das aplicações que têm despertando interesse é a utilização de microdispositivos na produção de Biodiesel. O Biodiesel é um combustível renovável, utilizando como matéria prima óleos vegetais ou gorduras animais, contribuindo menos para o efeito estufa (Gerpen, 2005; Freedman et al., 1984). Ele pode ser produzido pela reação química de um óleo vegetal (triglicerídeos) ou gordura animal com um álcool de cadeia curta como o metanol ou etanol, na presença de um catalisador básico, ácido ou enzimático (Gerpen, 2005). Assim sendo, uma elevada mistura dos reagentes, resulta em uma maior conversão em biodiesel. Em razão disso, no presente trabalho foi realizada a análise numérica da mistura dos fluidos óleo de Pinhão Manso e Etanol nos micromisturadores T $(n=2)$, cruz $(n=3)$ e duplo-T $(n=4)$.

\section{Modelo Computacional}

A Figura 1 apresenta o micromisturador-T e a identificação das dimensões utilizadas. Todos os misturadores possuem secção transversal retangular e mesmas dimensões. O comprimento do canal de entrada (Le), distância entre a entrada no misturador até o ponto de mistura dos fluidos, foi de 400 $\mu \mathrm{m}$. A altura dos misturadores foi de $200 \mu \mathrm{m}$. A largura foi $100 \mu \mathrm{m}$ para o comprimento de entrada (a) e de $200 \mu \mathrm{m}$ para o canal de mistura (w). O comprimento total do canal de mistura ( $\left.\mathrm{Lm}_{\mathrm{m}}\right)$ foi de $35100 \mu \mathrm{m}$. O software ANSYS ICEM 14.0 foi utilizado para gerar as geometrias e as malhas numéricas (subdivisão da geometria/domínio em um número finito de volumes de controle) dos micromisturadores. Antes de se estudar a mistura dos fluidos foi realizado um teste de malha. Nesse teste, geram-se malhas numéricas com diferentes quantidades de volume de controle e observa-se a variação dos resultados obtidos. Os fluidos utilizados nesse teste foram o álcool e água a $40{ }^{\circ} \mathrm{C}$. Esses fluidos foram utilizados para poder-se comparar o teste de malha realizado nesse trabalho com outros 
encontrados na literatura, utilizando os mesmos programas e condições computacionais, visto que não foram encontrados estudos de mistura entre Pinhão Manso e Etanol. Todas as malhas foram tetraédricas não estruturas.

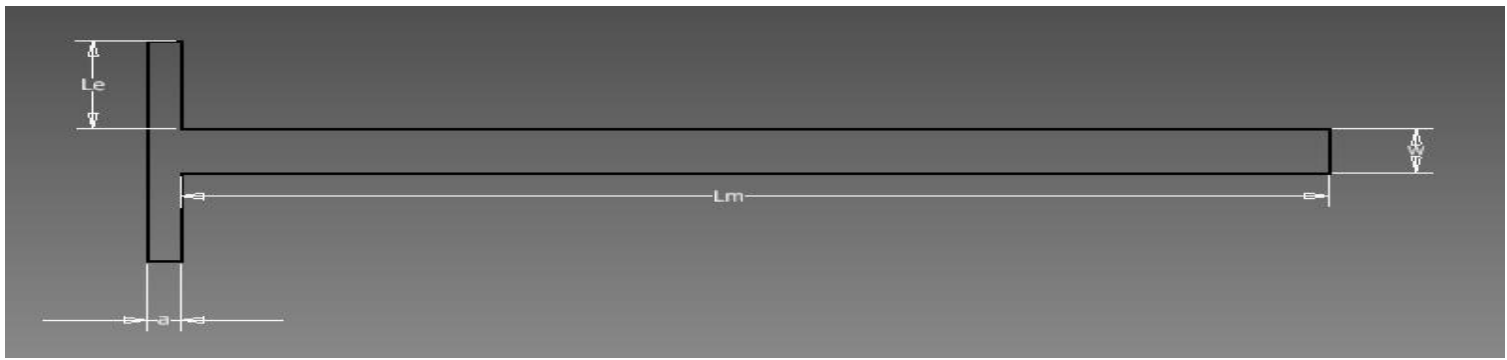

Figura 1. Micromisturador-T e a identificação das dimensões utilizadas nesse estudo.

As propriedades dos fluidos são apresentadas na Tabela 1 e os ácidos graxos presentes no óleo (informação utilizada para o cálculo da massa molecular do Pinhão Manso) na Tabela 2. Todas as análises numéricas foram realizadas na temperatura de $40{ }^{\circ} \mathrm{C}$. Para o estudo da mistura utilizou-se o número de Reynolds variando de 10 até 100.

Tabela 1- Propriedades físicas do óleo e do etanol.

\begin{tabular}{ccc}
\hline Fluidos & Densidade $\left(\mathbf{k g ~ m}^{\mathbf{- 3}}\right)$ & $\begin{array}{r}\text { Viscosidade cinemática } \\
(\mathbf{4 0} \mathbf{~} \mathbf{C})\left(\mathbf{m}^{\mathbf{2}} \mathbf{~ s}^{\mathbf{- 1}}\right)\end{array}$ \\
\hline Pinhão Manso & 911.2 & $3.482 \mathrm{e}-5$ \\
Etanol & 789 & $1.50 \mathrm{e}-6$ \\
\hline
\end{tabular}

Tabela 2 - Composição em ácidos graxos do óleo de Pinhão Manso (Souza 2010; Tapanes et al., 2008)

\begin{tabular}{ll}
\hline Ácidos Graxo & Percentagem molar \\
\hline Mirístico & $0.07-0.38$ \\
Palmítico & $11-16$ \\
Palmitoléico & $1-3.5$ \\
Esteárico & $5.3-7$ \\
Oléico & $38.23-43.5$ \\
Linoléico & $33-40$ \\
Araquídico & 0.2 \\
Gadoléico & 0.12 \\
Linolênico & $>0.8$ \\
\hline
\end{tabular}

O software utilizado para o estudo fluidodinâmico foi o ANSYS CFX-14.0 (ANSYS, 2009). Esse programa de dinâmica de fluidos de propósito geral resolve as equações de massa e momento utilizando o método dos volumes finitos. O ANSYS CFX consegue modelar a mistura de fluidos de muitos componentes físicos separados, no qual cada componente possui seu conjunto de propriedades 
físicas (Ansari e Kim, 2009). Por padrão o ANSYS CFX faz a suposição que os componentes formam uma mistura ideal, ou seja, as propriedades da mistura podem ser calculadas diretamente das propriedades dos componentes e suas proporções na mistura (ANSYS, 2012). O comportamento dos fluidos em macrocanais é descrito pela conservação da massa, energia e equilíbrio de forças, assumindo que o fluido pode ser considerado como contínuo. Em microescala, os fluidos, líquidos em especial, podem ser considerados contínuos, também. Nguyen e Wereley (2006) aponta para o fato que em um canal de $10 \mu \mathrm{m}$ pode haver 30000 moléculas de água, número suficiente para considerar o escoamento como contínuo. Nesses casos, as equações de transporte podem ser descritas pela equação da continuidade (Equação 1) e pelas equações de Navier-Stokes (Equação 2):

$$
\begin{aligned}
& \frac{\partial \rho}{\partial t}+\nabla(\rho \vec{u})=0 \\
& \rho \frac{\partial \vec{u}}{\partial t}+\rho(\nabla \vec{u}) \vec{u}=-\nabla p+\mu(\vec{u} \nabla \vec{u})+\rho f
\end{aligned}
$$

Em que u é a componente da velocidade, $\mu$ é a viscosidade, $\rho$ é a densidade, $\mathrm{p}$ é a pressão e $f$ caracteriza uma força externa.

Adotou-se para todos os casos simulados, esquema de alta resolução para os termos advectivos. Para calcular as velocidades de entrada utilizadas como condições de contorno para ambos os fluidos correspondendo aos números de Reynolds especificados, usou-se as dimensões do canal de mistura e as propriedades do óleo vegetal (viscosidade). A pressão estática foi estabelecida como zero na saída e condição de não-escorregamento foi aplicado nas paredes sólidas. Um critério de convergência RMS (Root mean square) de $1 \times 10^{-5}$ foi estabelecido, e um número de iterações foi definido entre 500 e 5000. As simulações foram resolvidas em processamento paralelo. Os nós computacionais são compostos por 8 processadores Intel Xeon de $3 \mathrm{GHz}$ com 16 GB de RAM, os quais usam o sistema operacional Linux de 64 bits.

O índice de mistura foi calculado com base no desvio padrão da fração mássica do óleo no micromisturador em uma secção transversal normal a direção do escoamento de acordo com a Equação 3:

$$
\sigma=\sqrt{\frac{\sum\left(x_{i}-\bar{x}\right)^{2}}{N}}
$$

Em que $\sigma$ é a variação da fração mássica, $x_{i}$ é a fração mássica no ponto de amostragem i, $\bar{x}$ é a média e $\mathrm{N}$ é o número de pontos de amostragem contidos na secção transversal. Em cada plano, $\mathrm{N}$ excedeu o número de 400 pontos de amostragem e esses planos foram equidistantes entre si. A eficiência da mistura entre o óleo vegetal e etanol foi calculada utilizando o parâmetro apresentado na Equação 4: 


$$
M=1-\sqrt{\frac{\sigma^{2}}{\sigma_{\text {máx }}^{2}}}
$$

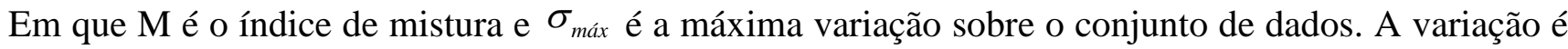
máxima para fluidos não misturados e mínima para misturados.

\section{RESULTADOS E DISCUSSÕES}

Na Figura 2 é apresentado a análise do teste de malha com diferentes volumes de controle que variaram de $1,80 \times 10^{5}$ a $1,43 \times 10^{6}$. O índice de mistura foi utilizado para avaliar as malhas numéricas. O teste foi realizado para o misturador-T com Reynolds igual a 100. Observa-se uma significativa variação do índice de mistura com o aumento do número de volumes de controle, sendo que na medida que a malha vai ficando mais refinada, o índice de mistura vai diminuindo. Para malhas mais refinadas (volumes de controle acima de $9,00 \times 10^{5}$ ), a mistura dos fluidos começa a tender para um valor em torno de 0,7. Trabalhos como os de Alam et al., (2013), Alam e Kim (2012) e Ansari e Kim (2009) que também realizaram um teste de malha com água e etanol, encontraram a mesma relação entre o refinamento da malha e o valor do índice de mistura. Por isso o valor de 1,14 $\times 10^{6}$ foi selecionado para o estudo da mistura nos micromisturadores, visto que os valores de $9,65 \times$ $10^{5}$ e $1,43 \times 10^{6}$ apresentaram comportamento diferente do esperado. Seria necessário mais testes com malhas de maior grau de refinamento, o que será considerado em publicações futuras.

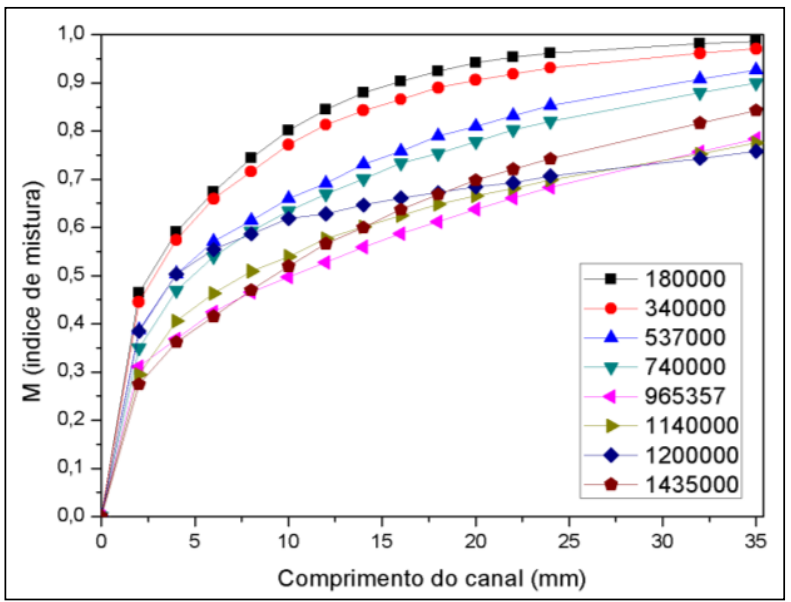

Figura 2. Teste de malha com diferentes números de volume de controle (Micromisturador- $\mathrm{T}$ e $\mathrm{Re}=$ 100)

O índice de mistura em função do número de Reynolds na saída dos micromisturadores é apresentado na Figura 3. Pode-se visualizar que o micromisturador em forma de cruz apresentou os melhores índices de mistura, com valores médio de 0,99. No misturador-T, o índice de mistura elevou-se com o aumento do número de Reynolds, sendo que o duplo-T possuiu um comportamento inverso, ou seja, a mistura diminui com o aumento da velocidade. É importante ressaltar que em 
baixos números de Reynolds (<50) o duplo-T realiza melhor a mistura dos fluidos do que o T. Como dito anteriormente, o escoamento de fluidos em microcanais tende preferencialmente ser laminar devido aos baixos número de Reynolds, dessa maneira a mistura das espécies ocorre apenas pelo mecanismo de difusão molecular. Entretanto, com o aumento da velocidade de escoamento, começa a ocorrer outro mecanismo de mistura das espécies: a advecção. A advecção causa uma perturbação no escoamento, aumentando a mistura das espécies (Alam e Kim, 2012). Isso pode explicar o porquê do índice de mistura aumentar com a elevação do Reynolds no misturador-T. Também se observa que a partir de um Reynolds de 55, o valor do índice de mistura permanece praticamente constante para esse misturador.

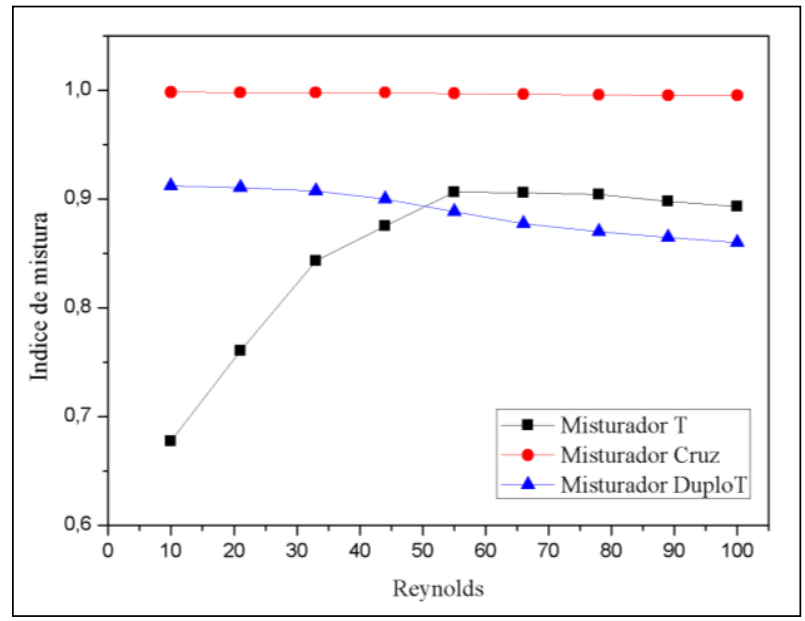

Figura 3. Variação do índice de mistura em função do número de Reynolds.

Ao aumentar a corrente de entrada de $n=2$ para 3 ou 4 subcorrentes diminui-se a largura da corrente de escoamento, aumentando a superfície de contato e reduzindo o caminho de mistura, elevando o índice de mistura. No caso de $n=3$, além da largura da corrente de escoamento ser menor, o óleo escoando no meio das correntes de álcool, permite que as moléculas de triglicerídeos difundam em duas direções ao invés de uma (Figura 4). O aumento da superfície de contato também ocorre no duplo-T (Em Reynolds igual a 10 o índice de mistura é igual a 0,91 contra 0,67 do T), porém com o aumento da velocidade ocorre a diminuição da mistura dos fluidos. Isso pode ser devido a diminuição da quantidade de óleo nesse misturador com o aumento da velocidade. Como as condições de contorno da velocidade foram iguais para todas as entradas, o óleo tendo uma viscosidade maior, possui uma resistência mais elevada ao movimento, assim o álcool move-se mais rápido preenchendo o canal de mistura com uma quantidade maior desse fluido. A Figura 5 mostra as linhas de corrente no Duplo-T, para um Reynolds de 10 e 100. Como é possível visualizar na figura, a diferença de velocidade entre os fluidos é mais acentuada quando o número de Reynolds é igual a 100. 


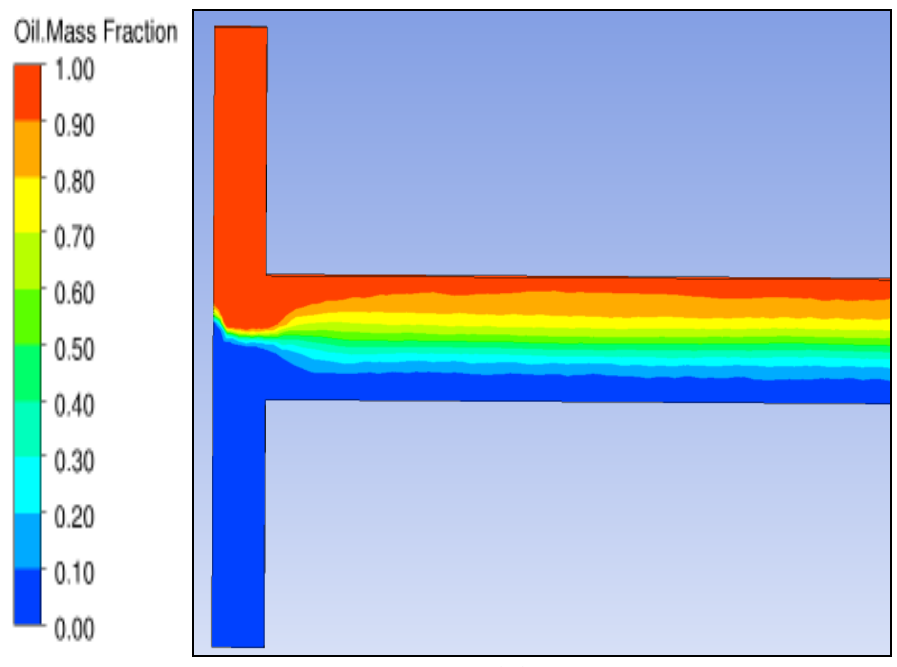

(a)

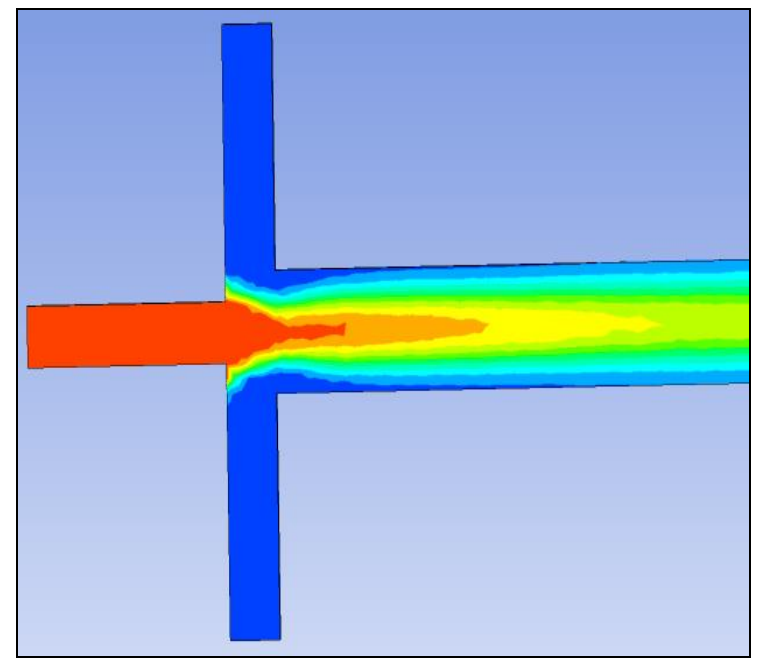

(b)

Figura 4. Distribuição da fração mássica dentro do canal de mistura (Re=10): (a) T e (b) Cruz.

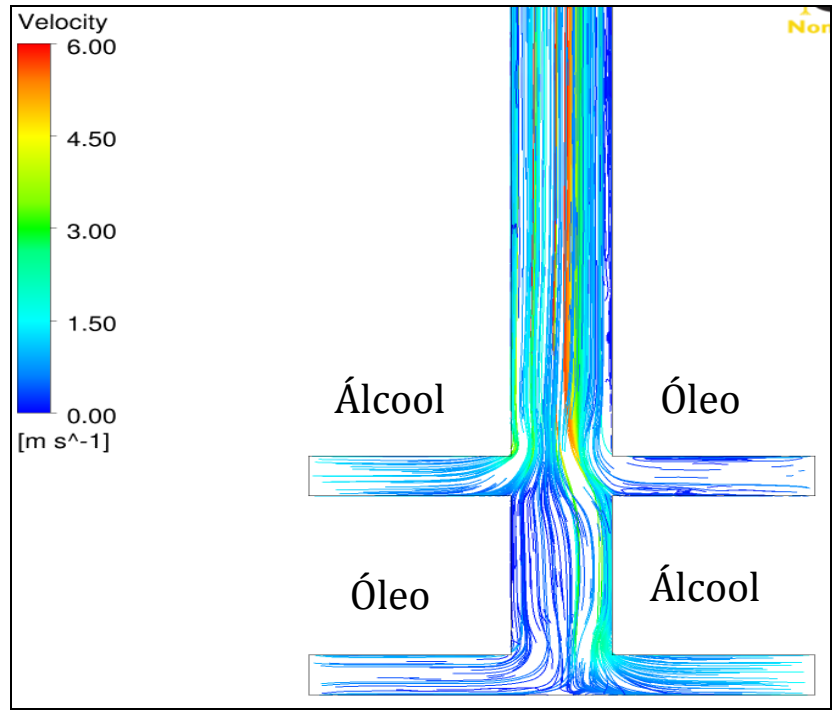

(a)

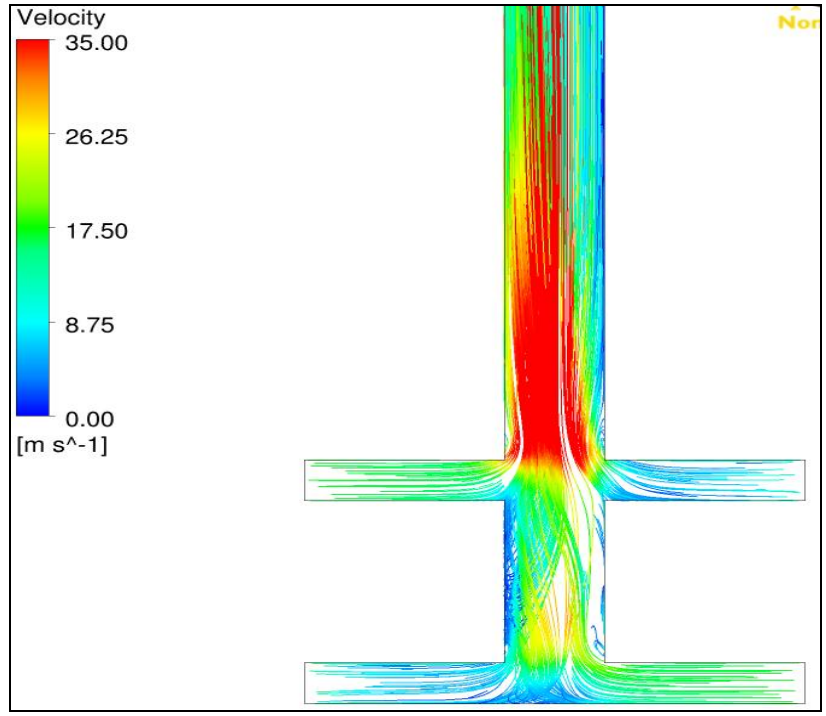

(b)

Figura 5. Linhas de corrente no micromisturador Duplo-T: (a) $\operatorname{Re}=10$ e (b) $\operatorname{Re}=100$.

\section{CONCLUSÃO}

A mistura entre o óleo de Pinhão Manso e etanol foi estudada numericamente utilizando o conceito de índice de mistura. A mistura foi avaliada para o número de Reynolds que variou de 10 a 100. Todos os micromisturadores ( $\mathrm{T}$, cruz e duplo $\mathrm{T}$ ) mostraram ótimos índices de mistura sendo o misturador em forma de Cruz apresentando os melhores resultados. O aumento do número de Reynolds elevou a mistura no misturador T, enquanto diminui no Duplo-T. A eficiência da mistura praticamente não é alterada pelo número de Reynolds no micromisturador em forma de Cruz. 


\section{AGRADECIMENTOS}

À FAPESP e CAPES pelo suporte financeiro.

\section{REFERÊNCIAS}

ALAM, A.; AFZAL, A.; KIM, K-Y. Mixing performance of a planar micromixer with circular obstruction in a curved microchannel. Chem Eng Res Des, v. 92, p. 423-434, 2013

ALAM, A.; KIM, K-Y. Analysis of mixing in a curved microchannel with rectangular grooves. Chem Eng J, v.181-182, p. 708-716, 2012.

ANSARI, M. A.; KIM, K-Y. A Numerical Study of Mixing in a Microchannel with Circular Mixing Chambers. Am Inst Chem Eng, v.55, p. 2217-2225, 2009.

ANSYS. ANSYS CFX-Solver Theory Guide.2009.

ANSYS. ANSYS CFX-Solver Modeling Guide.2012.

FREEDMAN, B.; PRYDE, E. H.; MOUNTS, T. L. Variables affecting the yields of fatty esters from transesterified vegetable oils. J Am Oil Chem Soc, v. 61, p.138-1643, 1984.

GERPEN, J. V. Biodiesel processing and production. Fuel Process Technol, v. 86, p. 1097- 1107, 2005.

GOULET, M-A.; KJEANG, E. Co-laminar flow cells for electrochemical energy conversion. J Power Sources, v. 260, p. 186-196, 2014.

MANSUR, A. E.; MINGXING, Y.; YUNDONG, W.; YOUYUAN, D. A State-of-the-Art Review of Mixing in Microfluidic Mixers. Chin J Chem Eng, v. 16, p. 503-516, 2008.

NGUYEN, N-T.; WU, Z. Micromixers- a review. J Micromech Microeng, v. 15, p. 1-16, 2005.

NGUYEN, N-T; WERELEY. S. T. Fundamentals and applications of microfluidics. Boston/London: Editora Artech House, 2006.

SOUZA, L. T. de A. Síntese enzimática do biodiesel de Jatropha curcas pela rota etílica. 2010. Dissertação (Mestre em Ciências) - Universidade de São Paulo, São Paulo, 2010.

TAPANES, N. C. O.; ARANDA, D. A. G.; CARNEIRO, J. W. de M.; ANTUNES, A. C. A. Transesterification of Jatropha curcas oil glycerides: Theoretical and experimental studies of biodiesel reaction. Fuel, v. 87, p. 2286-2295, 2008.

WHITESIDES, G. M. The origins and the future of microfluidics. Nature, v. 442, p. 368-373, 2006. 